\title{
Notes on a semigroup related to the dicyclic group $\mathrm{Q}_{\mathrm{n}}$
}

\author{
M. R. Sorouhesh and C. M. Campbell
}

\begin{abstract}
We consider certain properties of the semigroup $S$ defined by the presentation

$$
S=\left\langle a, b: a^{2^{n-1}}=1, b^{2}=a^{2^{n-2}}, b a=a b^{2^{n-1}-1}\right\rangle,(n \geq 3) .
$$
\end{abstract}

\section{Introduction and Preliminary Facts}

The purpose of this paper is to investigate computationally some remarkable properties of a certain finitely generated semigroup. For the terminology and notation see $[4,5]$. We know that if $A$ is an alphabet and $A^{+}$denotes the free semigroup on $A$, then a semigroup presentation is a pair $\langle A: R\rangle$ where $R \subseteq A^{+} \times A^{+}$. The elements of $A$ are called generators, and the elements of $R$ are relations. Some preliminaries and more information on semigroup presentations may be found in $[3,10]$. However, there are many semigroup presentations that each of which has some specific properties $[1,10,11]$.

The dicyclic group $\mathbf{Q}_{\mathbf{n}}$ is given by the presentation

$$
\left\langle a, b: a^{2^{n-1}}=1, b^{2}=a^{2^{n-2}}, b a=a b^{2^{n-1}-1}\right\rangle,
$$

where $n \geq 3$. We are interested here in the semigroup defined by the above presentation and so consider the following semigroup modification of it:

$$
S=\left\langle a, b: a^{2^{n-1}+1}=a, b^{2}=a^{2^{n-2}}, b a=a b^{2^{n-1}-1}\right\rangle, \quad(n \geq 3)
$$

Key Words: Finitely presented semigroups, Special semigroups.

2010 Mathematics Subject Classification: $20 \mathrm{M} 05$.

Received: 01.09.2016

Accepted: 24.10 .2016 
For the semigroup $S$, some auxiliary algebraic properties can be verified inductively which we use throughout the paper. They show that the the semigroup $S$, as a non-group and non-commutative semigroup, is a concrete example of different kinds of semigroups.

Lemma 1.1. For every $k \in\{0\} \cup \mathbb{N}$ with $n \geq 3$ we have:

(a) if $i=4 k+1$ then $b^{\left(2^{n-1}-1\right) i}=a^{2^{n-2}} b$;

(b) if $i=4 k+2$ then $b^{\left(2^{n-1}-1\right) i}=a^{2^{n-2}}$;

(c) if $i=4 k+3$ then $b^{\left(2^{n-1}-1\right) i}=a^{2^{n-1}} b$;

(d) if $i=4 k+4$ then $b^{\left(2^{n-1}-1\right) i}=a^{2^{n-1}}$.

Proof. It is easy to see that modulo $2^{n-1}$ and for a positive integer $t$, the following trivial identities are satisfied:

$$
t 2^{n-1}-2^{n-2} \equiv 2^{n-2} \quad(1), \quad t 2^{n-1}-2^{n-1} \equiv 2^{n-1} .
$$

We prove only the assertion concerning the part $(a)$ and the remaining cases can be proved similarly. For $(a)$ use an inductive method on $k$. Let $k=0$ so we have:

$$
\begin{aligned}
b^{\left(2^{n-1}-1\right)} & =\left(b^{2}\right)^{\left(2^{n-2}-1\right)} \cdot b=\left(a^{2^{n-2}}\right)^{2^{n-2}-1} \cdot b \quad\left(\text { for } \quad b^{2}=a^{2^{n-2}}\right) \\
& =a^{\left(2^{2 n-4}-2^{n-2}\right)} \cdot b \stackrel{(1)}{=} a^{2^{n-2}} \cdot b .
\end{aligned}
$$

Assume $(a)$ is true for $k$, i.e.; $b^{\left(2^{n-1}-1\right)(4 k+1)}=a^{2^{n-2}} b$ then

$$
\begin{aligned}
a^{2^{n-2}} \cdot b & =a^{\left(2^{n-2}+2^{n-1}\right)} \cdot b \stackrel{(2)}{=} a^{2^{n-2}}\left(a^{2^{n-2}}\right)^{\left(2^{n}-2\right)} \cdot b=a^{2^{n-2}} \cdot\left(b^{2}\right)^{\left(2^{n}-2\right)} \cdot b \\
& =\left(a^{2^{n-2}} \cdot b\right) \cdot b^{2^{n+1}-4}=b^{\left(2^{n-1}-1\right)(4 k+1)} \cdot b^{2^{n+1}-4}=b^{\left(2^{n-1}-1\right)(4 k+5)} .
\end{aligned}
$$

Lemma 1.2. For $1 \leq i \leq 2^{n-1}$ we have $a^{i}=b a^{(i-1) 2^{n-2}+i} b$.

Proof. The result is true for $i=1$. Indeed, $b a b=\left(a b^{2^{n-1}-1}\right) \cdot b=a b^{2^{n-1}}=$ $a\left(b^{2}\right)^{2^{n-2}}=a\left(a^{2^{n-2}}\right)^{2^{n-2}}=a\left(a^{2^{2 n-4}}\right)=a$. If the claim is true for $i$ then the relations of $S$ and the first part of Lemma 1.1 gives:

$$
b a=a b^{2^{n-1}-1}=a\left(a^{2^{n-2}} b\right)=a^{2^{n-2}+1} b,
$$


and so $a^{i+1}=a^{i} \cdot a=\left(b a^{(i-1) 2^{n-2}+i} b\right) \cdot a=\left(b a^{(i-1) 2^{n-2}+i}\right) \cdot(b a)$ which by $(3)$ is equal to

$$
\left(b a^{(i-1) 2^{n-2}+i}\right) \cdot\left(a^{2^{n-2}+1} b\right)=b a^{i 2^{n-2}+(i+1)} b .
$$

As a result of the above lemma we have:

Corollary 1.3. In semigroup $S$ we have:

$$
a^{i} b=b a^{i\left(2^{n-2}+1\right)}, b a^{i}=a^{i\left(2^{n-2}+1\right)} b \quad\left(1 \leq i \leq 2^{n-1}\right) .
$$

Lemma 1.4. The semigroup $S$ may be partitioned as

$$
S=\{b\} \cup\left\{a^{i}, 1 \leq i \leq 2^{n-1}\right\} \cup\left\{b a^{j}, 1 \leq j \leq 2^{n-1}\right\} .
$$

Proof. By the corollary above and the relations of $S$, we conclude that the only words in $S$ starting with $a$ are exact powers of $a$.

Proposition 1.5. For elements $a$ and $b$ while $1 \leq i, j \leq 2^{n-1}$ the following relations hold:

(a) $\left(a^{i}\right)(b)=(b)\left(a^{i}\right) \cdot\left(b a^{2^{n-2}-i}\right) \cdot(b)\left(a^{i}\right)$;

(b) $\left(a^{i}\right)\left(a^{j}\right)=\left(a^{j}\right)\left(a^{i}\right) \cdot\left(a^{2^{n-2}-(i+j)}\right) \cdot\left(a^{j}\right)\left(a^{i}\right)$;

(c) $\left(a^{i}\right)\left(b a^{j}\right)=\left(b a^{j}\right)\left(a^{i}\right) \cdot\left(b a^{2^{n-2}(1+j)-(i+j)}\right) \cdot\left(b a^{j}\right)\left(a^{i}\right)$;

(d) $(b)\left(a^{i}\right)=\left(a^{i}\right)(b) \cdot\left(a^{2^{n-2}-i} b\right) \cdot\left(a^{i}\right)(b) ;$

(e) $(b)\left(b a^{i}\right)=\left(b a^{i}\right)(b) \cdot\left(a^{2^{n-2}-i}\right) \cdot\left(b a^{i}\right)(b)$;

(f) $(b)(b)=(b)(b) \cdot\left(b^{2}\right) \cdot(b)(b) ;$

(g) $\left(b a^{j}\right)\left(a^{i}\right)=\left(a^{i}\right)\left(b a^{j}\right) \cdot\left(b a^{\left(2^{n}-1\right)(i+j)+2^{n-2}}\right) \cdot\left(a^{i}\right)\left(b a^{j}\right)$;

(h) $\left(b a^{i}\right)(b)=(b)\left(b a^{i}\right) \cdot\left(b a^{\left.2^{n-1}+i\left(2^{n-2}-2\right)\right)}\right) \cdot(b)\left(b a^{i}\right)$;

(k) $\left(b a^{i}\right)\left(b a^{j}\right)=\left(b a^{j}\right)\left(b a^{i}\right) \cdot\left(a^{2^{n-2}(1+i)-(i+j)}\right) \cdot\left(b a^{j}\right)\left(b a^{i}\right)$;

(l) $\left(a^{i}\right)\left(a^{2^{n-1}-i}\right)=\left[\left(a^{i}\right)\left(a^{2^{n-1}-i}\right)\right]^{2}$;

(m) $(b)\left(b^{3}\right)=\left[(b)\left(b^{3}\right)\right]^{2}$;

(n) $\left(b a^{i}\right)\left(b a^{i\left(2^{n-2}-1+2^{n-2}\right.}\right)=\left[\left(b a^{i}\right)\left(b a^{i\left(2^{n-2}-1+2^{n-2}\right.}\right)\right]^{2}$. 
Proof. We start from the right hand side of $(a)$. Corollary 1.3 gives:

$$
(b)\left(a^{i}\right)\left(b a^{2^{n-2}-i}\right)(b)\left(a^{i}\right)=\left(a^{i\left(2^{n-2}+1\right)} b\right) \cdot\left(b a^{2^{n-2}-i}\right) \cdot\left(a^{i\left(2^{n-2}+1\right)} b\right)
$$

which is equal to $a^{i \times 2^{n-2}+i+2^{n-2}+2^{n-2}-i+i \times 2^{n-2}+i} b$ and by (2) we get $a^{i} b$ as desired. For $(c)$ Corollary 1.3 and the relations of $S$ yield $\left(b a^{j}\right)\left(a^{i}\right)=$ $a^{\left(1+2^{n-2}\right)(i+j)} b$ and so

$$
\left(b a^{j}\right)\left(a^{i}\right)\left(b a^{2^{n-2}(1+j)-(i+j)}\right)\left(b a^{j}\right)\left(a^{i}\right)=a^{i} \cdot a^{j\left(2^{n-2}+1\right)} b=\left(a^{i}\right)\left(b a^{j}\right) .
$$

Rewriting the right hand side of $(d)$ gives $(b)\left(a^{i\left(2^{n-2}+1\right)}\right)\left(b a^{i} b\right)$ which is equal to $b\left(a^{i\left(2^{n-2}+1\right)}\right)\left(a^{i\left(2^{n-2}+1\right)-i}\right)$, which is the left part of $(c)$. For $(e)$ we have $\left(b a^{i}\right)(b)\left(a^{2^{n-2}-i}\right)\left(b a^{i}\right)(b)=\left(a^{i\left(2^{n-2}+1\right)+2^{n-2}}\right)\left(a^{2^{n-2}-i}\right)\left(a^{i\left(2^{n-2}+1\right)+2^{n-2}}\right)$

$$
=a^{i+2^{n-2}}=(b)\left(b a^{i}\right) .
$$

Since by Corollary 1.3, $a^{i}\left(b a^{j}\right)=a^{i+j\left(2^{n-2}+1\right)}$, the right hand side of $(g)$ can be simplified as

$$
\left(b a^{j}\right)\left(a^{i\left(2^{n-2}+1\right)}\right)\left(a^{2^{n-2}+1\left[\left(2^{n-2}-1\right)(i+j)+2^{n-2}\right]}\right) a^{i}\left(b a^{j}\right),
$$

which is equal to

$$
\left(b a^{j}\right)\left(a^{i\left(2^{n-2}+1\right)+2^{n-2}-(i+j)}\right)\left(a^{2^{n-2}+1} b^{2} a^{j}\right)=\left(b a^{j}\right)\left(a^{i}\right) .
$$

Similarly $\left(b a^{j}\right)\left(b a^{i}\right)=a^{j \times\left(2^{n-2}+1\right)+2^{n-2}+i}$ by Corollary 1.3 and the right hand side of $(k)$ is reduced as $a^{2^{n-2}+i \times 2^{n-2}+i+j}=\left(b a^{i}\right)\left(b a^{j}\right)$ which shows that $(k)$ is valid. By using (1), (2) and (3), the proofs for $(b),(l)$ and $(n)$ are routine and considering the relations of $S ;(f),(h)$ and $(m)$ can be easily verified.

Proposition 1.6. For every $x, y \in S$ we have

$$
x y=a^{s}, y x=a^{r}, \quad \text { or } \quad x y=b a^{s}, y x=b a^{r},
$$

where $1 \leq s, r \leq 2^{n-1}$ and $r \equiv s$ modulo $2^{n-2}$.

Proof. The proof is similar to the proof of Proposition 1.5 by taking possible forms of $x$ and $y$ of $S$. Firstly we note that, if $r \equiv s$ modulo $2^{n-2}$ then $s=r-k \times 2^{n-2}$ where $k \in \mathbb{Z}^{+}$and so for an element $a \in S$ we have:

$$
a^{s}=a^{r-k \times 2^{n-2}}=a^{r-k \times 2^{n-2}+k \times 2^{n-1}}=a^{r+k \times 2^{n-2}} .
$$

And then, by the relations of $S$, all $x y$ have forms $a^{s}$ or $b a^{s}$ where $1 \leq s \leq 2^{n-1}$ and none of them ends in $b$. When $x y=a^{s}$, we have the following possible cases: 
(a) $x=a^{i}, y=a^{j}$;

(b) $x=b a^{i}, y=b a^{j}$;

(c) $x=b, y=b$;

(d) $x=b, y=b a^{i}$;

(e) $x=b a^{i}, y=b$.

In parts $(a)$ and $(c)$ we have $x y=a^{i+j}=y x$ and $x y=a^{2^{n-2}}=y x$ respectively so they are obviously satisfied. If $x=b a^{i}, y=b a^{j}$ where $1 \leq i, j \leq 2^{n-1}$ so by using Corollary 1.3 we have:

$$
y x=a^{s}=a^{j\left(1+2^{n-2}\right)+i+2^{n-2}+i \times 2^{n-1}}=a^{r}=x y \cdot a^{(i+j) 2^{n-2}},
$$

where $s=j\left(1+2^{n-2}\right)+i+2^{n-2}$ and $r=i\left(1+2^{n-2}\right)+j+2^{n-2}+(i+j) 2^{n-2}$ respectively. Hence, $r \equiv s$ modulo $2^{n-2}$ and so $(b)$ is true. The proof for parts $(d)$ and $(e)$ is similar and we check just part $(d)$. Let $x=b$ and $y=b a^{i}$ for some $1 \leq i \leq 2^{n-1}$. Then we get $y x=a^{i\left(1+2^{n-2}\right)+2^{n-2}}=a^{i+2^{n-2}} \times a^{i \times 2^{n-2}}=$ $x y \times a^{i \times 2^{n-2}}$ which shows that the claim is valid for $(d)$. Now, for elements $x, y \in S, x y=b a^{s}\left(1 \leq s \leq 2^{n-1}\right)$. So we have the cases below:

(f) $x=a^{i}, y=b a^{j}$;

(g) $x=a^{i}, y=b$;

(h) $x=b a^{j}, y=a^{i}$;

(m) $x=b, y=a^{i}$.

The claims in parts $(f)$ and $(h)$ are proved similarly. The same is true when considering $(g)$ and $(m)$ so we need to check the validity of the proposition just in parts $(f)$ and $(g)$. For $(f)$ we have $y x=b a^{i+j}=b a^{r}$ and $x y=b a^{r+i \times 2^{n-2}}=$ $b a^{s}$ where $r \equiv s$ modulo $2^{n-2}$. In $(g)$ :

$$
x y=a^{i} b=b a^{i\left(1+2^{n-2}\right)}=b a^{s}, \quad y x=b a^{i}=b a^{r},
$$

which shows that $r \equiv s$ modulo $2^{n-2}$. This completes the proof.

Proposition 1.7. In the semigroup $S$ and for every elements $x, y$ and $z$ we have $x y z y x=y x z x y$. 
Proof. Let $x, y \in S$. According to the previous proposition, we can consider two cases for $x y$, i.e.; $x y=a^{s}\left(y x=a^{r}\right)$ or $x y=b a^{s}\left(y x=b a^{r}\right)$ where $1 \leq s \leq 2^{n-1}$ and $r \equiv s$ modulo $2^{n-2}$. Suppose $z \in S$ and $x y=a^{s}$. If for some $1 \leq i \leq 2^{n-1}, z=a^{i}$ then $x y z y x=a^{s+i+r}=y x z x y$. If for some $1 \leq i \leq 2^{n-1}$, $z=b a^{i}$ then $x y z y x=a^{s} \cdot b a^{i} \cdot a^{r}=a^{r+k \times 2^{n-2}} \cdot b a^{i} \cdot a^{r}=a^{r} \cdot b a^{i+k \times 2^{n-2}\left(1+2^{n-2}\right)}$.' $a^{r}=a^{r} \cdot b a^{i} \cdot a^{s}=y x z x y$ for some $k \in \mathbb{Z}^{+}$. If $z=b$ then for some $k \in \mathbb{Z}^{+}$ we get $x y z y x=a^{s} b a^{r}=a^{r+k \times 2^{n-2}} b a^{r}=a^{r} b a^{k \times 2^{n-2}\left(1+2^{n-2}\right)} a^{r}=a^{r} b a^{s}$. For $z \in S$ and $x y=b a^{s}$, the proof is similar.

\section{Main results}

A semigroup $S$ is called commuting regular if for any $x, y \in S$ there exists $z \in S$ such that $x y=y x z y x$. If for any $x \in S$ there exists $y \in S$ such that $x y=(x y)^{2}$, then $S$ is called E-inversive [2]. Whenever for all $x, y$ and $z$ of $S$ we have $x y z y x=y x z x y$ then $S$ is known as a $C_{2}-$ semigroup [9].

Theorem 2.1. Let $n \geq 3$. The semigroup

$$
S=\left\langle a, b: a^{2^{n-1}+1}=a, b^{2}=a^{2^{n-2}}, b a=a b^{2^{n-1}-1}\right\rangle,
$$

is a finite non-abelian commuting regular and E-inversible semigroup of order $2^{n}+1$. Moreover $S$ is a $C_{2}$ - semigroup.

Proof. It is enough to consider different cases for $x, y \in S$ as in Lemma 1.4. Then, considering the results of Proposition 1.5 yields the proofs of commuting regularity and E-inversibility respectively. Obviously, $S$ is a non-abelian semigroup of order $2^{n}+1$. For the rest we consider Proposition 1.7.

\section{Remark 1.}

When $n=3$, by Lemma 2.3. of [11], we showed that the semigroup:

$$
S=\left\langle a, b: a^{5}=a, b^{2}=a^{2}, b a=a b^{3}\right\rangle=\left\{a, b, a^{2}, a^{3}, a^{4}, a b, a^{2} b, a^{3} b, a^{4} b\right\}
$$

is also a quasi-commutative semigroup of order 9 .

Lemma 2.2. For $n \geq 3$ all elements of $S$ except for $b$ are regular. Moreover $S$ is a $\pi$-regular semigroup.

Proof. The relations of $S$ show $b$ is an indecomposable element so it cannot be regular. For the other cases, we may consider the following points which can be verified easily:

$$
a^{i}=a^{i} \cdot\left(a^{2^{n-1}-i}\right) \cdot a^{i}, \quad b a^{i}=b a^{i} \cdot\left(b a^{2^{n-2}(i+1)-i}\right) \cdot b a^{i} \quad\left(1 \leq i \leq 2^{n-1}\right)
$$


Also, part (f) of Proposition 1.5 and the later equalities yield

$$
b^{2} \in b^{2} S b^{2}, \quad x \in x S x,
$$

for all $x(\neq b) \in S$. Therefore the semigroup $S$ is $\pi$-regular.

An idempotent $e \in S$ is called primitive whenever $f \in E(S)$ and $f=e f=$ $f e$ then we have $f=e$. If in a semigroup $S$ all idempotents are primitive then the semigroup is named primitive.

Theorem 2.3. For $n \geq 3$, the only idempotent of $S$ is $e=b^{4}$ and so $S$ is primitive.

Proof. As $\left(a^{2^{n-1}}\right)^{2}=a^{2^{n}}=a^{2^{n-1}\left(2^{n-1}+1\right)}=a^{2^{n-1}}$ where $n \geq 3$ so $a^{2^{n-1}}=b^{4}$ is an idempotent. Since $b$ is not regular it cannot be an idempotent. This shows that:

$$
\left(b a^{i}\right)^{2}=a^{i\left(2^{n-1}+1\right)+2^{n-2}+i} \neq b a^{i} .
$$

Indeed, $b \notin\langle a\rangle$. Therefore $E(S)$ is a singleton and so $S$ is primitive.

Corollary 2.4. For $n \geq 3, e S e$ is a unipotent monoid. In fact $S$ is a unipotent semigroup and so it is a power joined semigroup.

Proof. As a consequence of the previous theorem and Corollary 1 [2] eSe is a unipotent monoid. For the rest we may consider [7].

Lemma 2.5. For $n \geq 3, S^{2}=S-\{b\}$ is a unique proper maximal ideal of $S$. Moreover $S^{2}=[a]$ in which $[a]$ is the principle ideal of $S$ generated by $a$.

Proof. Regarding Lemma 2.2 and that $S^{2} \varsubsetneqq S$, we have $S^{2}=S-\{b\}$ so it is a maximal ideal of $S$. Obviously for any other proper maximal ideal $N$ of $S$, $b \notin N$ and so $N \subseteq S^{2}$ and so $N=S^{2}$. By the identities 1.3 and Lemma 2.2, $S^{2} \subseteq[a]$ and so the proof is complete.

Remark 2. Since $b^{2} \in S^{2}$ so, element $b$ would be a nilpotent with respect to $[a][6]$.

A regular semigroup $S$ is called a Clifford semigroup if all idempotent elements of $S$ are central [8].

Corollary 2.6. For $n \geq 3, S^{2}$ is a Clifford semigroup.

Proof. Obviously, $e=b^{4} \in S^{2}$ is central.

A semigroup $S$ is abundant if every minimal ideal of $S$ contains an idempotent element. 
Corollary 2.7. For $n \geq 3, S$ is an abundant semigroup.

Proof. Since every minimal ideal of $S$ necessarily contains the only idempotent $b^{4} \in S$ so the semigroup is abundant.

Lemma 2.8. For $n \geq 3, S=[b]$ where $[b]$ is the principle ideal of $S$ generated by $b$.

Proof. Using Lemma 1.2 and identities 1.3 the proof is clear.

Corollary 2.9. For $n \geq 3, S$ has exactly two of classes.

Proof. Since $S^{2}=S-\{b\}$ is a regular proper subsemigroup of $S$ so

$$
[b]_{\mathcal{J}} \cap S=\{b\}, \quad[a]_{\mathcal{J}} \cap S=S^{2}=[a] .
$$

\section{References}

[1] A. Arjomandfar, C. M. Campbell and H. Doostie, Semigroups related to certain group presentations, Semigroup Forum $\mathbf{8 5}$ (2012), no. 3, 533-539.

[2] S. Bogdanovic, M. Ciric, A. Stamenkovic, Primitive idempotents in semigroups, Mathematica Moravica 5 (2001), 7-18.

[3] C. M. Campbell, E. F. Robertson, N. Ruskuc, R .M. Thomas, Semigroup and group presentations, Bull. London Math. Soc. 27 (1995), 46-50.

[4] A. H. Clifford and G. B. Preston, The algebraic theory of semigroups I, Amer. Math. Soc. (1961).

[5] J. M. Howie, An introduction to semigroup theory, Academic Press Inc, (1967).

[6] F. Kmet On radicals in semigroups, Math. Slovaca, 32, no. 2 (1982), 183-188.

[7] R. G. Levin, T. Tamura, Notes on commutative power joined semigroups, Pacific J. Math., 35, no. 3 (1970), 673-679.

[8] A. Nagy, Special Classes of Semigroup, Kluwer Academic Publishers, (2001). 
[9] B. H. Neumann, T. Taylor, Subsemigroups of Nilpotent Groups, Proc. Roy. Soc. Ser. A, 274 (1963), 1-4.

[10] E. F. Robertson and Y. Unlu, On semigroup presentations, Proc. Edinburgh Math. Soc. 236 (1993), no. 1, 55-68.

[11] M. R. Sorouhesh, H. Dosstie, Quasi-commutative semigroups of finite order related to Hamiltonian groups, Bull. Korean Math. Soc. 52 (2015), No. 1, 239-246.

Mohammad R. SOROUHESH,

Department of Mathematics,

Islamic Azad University,

South Tehran Branch, Tehran, Iran. Email: sorouhesh@azad.ac.ir

Colin M. CAMPBELL,

School of Mathematics and Statistics,

University of St. Andrews,

North Haugh, St. Andrews, Fife KY16 9SS, Scotland, UK.

Email: cmc@st-andrews.ac.uk 